\title{
Nonalcoholic fatty liver disease in type 2 diabetes: A bad omen. But can we do something to change it?
}

\author{
Fernando Bril \\ Division of Endocrinology, Diabetes and Metabolism, University of Alabama at Birmingham, Birmingham, AL 35249, USA. \\ Correspondence to: Dr. Fernando Bril, Division of Endocrinology, Diabetes and Metabolism, Department of Medicine, University \\ of Alabama at Birmingham, 510 20th Street South, FOT 755, Birmingham, AL 35249, USA. E-mail: fbril@uab.edu \\ How to cite this article: Bril F. Nonalcoholic fatty liver disease in type 2 diabetes: A bad omen. But can we do something to
change it? Metab Target Organ Damage 2021;1:5. https://dx.doi.org/10.20517/mtod.2021.06
}

Received: 14 Jul 2021 Accepted: 12 Aug 2021 Available online: 18 Aug 2021

Academic Editor: Amedeo Lonardo Copy Editor: Xi-Jun Chen Production Editor: Xi-Jun Chen

In an article published in Diabetes Care last year, Younossi et al. ${ }^{[1]}$ constructed different models to estimate the economic and clinical implications of nonalcoholic fatty liver disease (NAFLD) in patients with type 2 diabetes (T2D). It was a provocative study, which allowed into perspective the real extent of the NAFLD epidemic in patients with $\mathrm{T} 2 \mathrm{D}$ and the serious consequences that these patients face. Their results were yet another forewarning that this is a growing problem and that there is an urgent need to change the natural history of millions of patients with NAFLD and T2D.

Based on their prediction, over the next 20 years in the United States, there will be 65,000 liver transplants, 812,000 liver-related deaths, and 1.37 million deaths due to cardiovascular events in patients with NAFLD and T2D. For their modeling, the authors considered an "incident population" (i.e., newly diagnosed) and a "prevalent population" (i.e., already diagnosed). As expected, among patients with newly diagnosed NAFLD, liver-related mortality and liver-related complications were not as significant as cardiovascular deaths. However, in patients with established NASH, the numbers of predicted liver-related and cardiovascular deaths were very similar (794,638 vs. 883,469, respectively). In light of these findings, the current dogma that NASH is exclusively under the umbrella of hepatologists should be revisited. These patients need a multidisciplinary approach, not only focusing on their livers but also targeting hyperglycemia, dyslipidemia, and other cardiovascular risk factors. Therefore, primary care physicians, endocrinologists, and any other providers treating patients with $\mathrm{T} 2 \mathrm{D}$ are at center stage. Indeed, most of the

(c) The Author(s) 2021. Open Access This article is licensed under a Creative Commons Attribution 4.0 International License (https://creativecommons.org/licenses/by/4.0/), which permits unrestricted use, sharing, adaptation, distribution and reproduction in any medium or format, for any purpose, even commercially, as long as you give appropriate credit to the original author(s) and the source, provide a link to the Creative Commons license, and indicate if changes were made. 
medications with proven histological efficacy in randomized, controlled trials come from the diabetes world (i.e., pioglitazone, semaglutide), and these providers may be more comfortable than hepatologists prescribing them.

From an economic standpoint, Younossi et al. ${ }^{[1]}$ estimated that during the next 20 years, these patients would cost the healthcare system $\$ 1672$ billion, with a total cost per person per year of $\$ 7700$. While most of these costs were attributed to diabetes care, with only $\sim 11 \%$ attributed to NAFLD-related costs, in patients with established NASH, NAFLD-related costs represented a shocking $25 \%$ of all costs. These costs are remarkable, especially if we consider that the USA's overall per capita health care expenditure in 2019 was estimated at $\$ 11,582$, according to a report published by Centers for Medicare \& Medicaid Services ${ }^{[2]}$.

Due to the significant number of assumptions, these predictions are likely inaccurate. However, the message this article delivers is still loud and clear: we have a growing problem with a substantial clinical and economic impact, and this should be evident to the whole health care system, regardless of the exact numbers. Nevertheless, these predictions have not been written in stone. Therefore, the question that we should be asking ourselves as health care providers is what we can do to change the natural history of this disease.

In the last few decades, we have learned a considerable amount regarding the epidemiology, pathophysiology, diagnosis, and treatment of NAFLD and NASH. Despite this, patients with NAFLD and $\mathrm{T} 2 \mathrm{D}$ continue to go undiagnosed and untreated for long periods of time $\mathrm{e}^{[3]}$, oftentimes presenting to their providers directly with decompensated cirrhosis. Unfortunately, the later this condition is diagnosed, the fewer the options to turn its course. Even in large randomized, controlled trials, the efficacy of current medications to regress liver fibrosis is almost negligible ${ }^{[4-7]}$. However, several of these medications did show efficacy inducing resolution of NASH in patients with $\mathrm{T}_{2} \mathrm{DM}^{[4,5,7]}$, with some even showing delay in the progression of fibrosis ${ }^{[4]}$. Therefore, early diagnosis and treatment of these patients are crucial if we do not want the predictions of Younossi et al. ${ }^{[1]}$ to become true. And even more important is focusing on the prevention of this disease.

The article of Younossi et al. ${ }^{[1]}$ did not devote much attention to the role of obesity in the progression of $\mathrm{NASH}$ or its influence on the clinical and economic complications of NASH. However, a large amount of evidence supports that the obesity epidemic is the fuel that feeds the fire of NASH and T2D. Indeed, NASH and T2D may just be two small tips of a huge iceberg, which has a solid base composed of obesity, sedentarism, and insulin resistance. Obstructive sleep apnea, polycystic ovary syndrome, hypertension, and dyslipidemia, which we usually face in the clinic, are other tips of this iceberg. The prevention of all these metabolic entities, including NASH, requires a multidisciplinary and systematic approach targeting weight loss. In recent years, we have seen a continuous increase in the use of bariatric surgery, with some newer techniques available. This, combined with new effective pharmacological options, such as the recently approved $2.4 \mathrm{mg}$ semaglutide weekly, provide new tools in our fight against obesity. Only with this strategy will we succeed in turning the course of this epidemic and making sure that Younossi's predictions do not come true.

\section{DECLARATIONS}

\section{Authors' contributions}

The author contributed solely to the article. 


\section{Availability of data and materials}

Not applicable.

\section{Financial support and sponsorship}

None.

\section{Conflicts of interest}

The author declared that there are no conflicts of interest.

\section{Ethical approval and consent to participate}

Not applicable.

\section{Consent for publication}

Not applicable.

\section{Copyright}

(c) The Author(s) 2021.

\section{REFERENCES}

1. Younossi ZM, Tampi RP, Racila A, et al. Economic and clinical burden of nonalcoholic steatohepatitis in patients with type 2 diabetes in the U.S. Diabetes Care 2020;43:283-9. DOI PubMed

2. Centers for Medicare \& Medicaid Services. National Health Expenditures 2019 Highlights. Available at: https:/www.cms.gov/files/document/highlights.pdf. [Last accessed on 17 Aug 2021].

3. Rinella ME, Lominadze Z, Loomba R, et al. Practice patterns in NAFLD and NASH: real life differs from published guidelines. Therap Adv Gastroenterol 2016;9:4-12. DOI PubMed PMC

4. Cusi K, Orsak B, Bril F, et al. Long-term pioglitazone treatment for patients with nonalcoholic steatohepatitis and prediabetes or type 2 diabetes mellitus: a randomized trial. Ann Intern Med 2016;165:305-15. DOI PubMed

5. Newsome PN, Buchholtz K, Cusi K, et al. A placebo-controlled trial of subcutaneous semaglutide in nonalcoholic steatohepatitis. $N$ Engl J Med 2021;384:1113-24. DOI PubMed

6. Younossi ZM, Ratziu V, Loomba R, et al; REGENERATE Study Investigators. Obeticholic acid for the treatment of non-alcoholic steatohepatitis: interim analysis from a multicentre, randomised, placebo-controlled phase 3 trial. Lancet 2019;394:2184-96. DOI PubMed

7. Sanyal AJ, Chalasani N, Kowdley KV, et al. Pioglitazone, vitamin E, or placebo for nonalcoholic steatohepatitis. $N$ Engl $J$ Med 2010;362:1675-85. DOI PubMed PMC 